\title{
Vigilância em saúde na pandemia da COVID-19: retomada das atividades eletivas em rede de hospitais de ensino
}

\author{
Health surveillance in the COVID-19 pandemic: resumption of \\ elective activities in the network of teaching hospitals
}

\section{Gabriela de Oliveira Silval,* (ID \\ Márcia Amaral Dal Sasso' \\ Leili Mara Mateus da Cunha' (D) \\ Tamela Beatriz Matinada da Silva' iD \\ Isaac Newton Machado Bezerra"I' (iD)}

Grasiela Piuvezam" iD
I Empresa Brasileira de Serviços Hospitalares (Ebserh), Brasília, DF, Brasil

" Programa de Pós-Graduação em Saúde Coletiva (PPGSCOL), Universidade Federal do Rio Grande do Norte (UFRN), Natal, RN, Brasil

III Universidade Federal de Pernambuco (UFPE), Vitória de Santo Antão, PE, Brasil

\footnotetext{
* E-mail: gabioliveira18@gmail.com
}

\begin{abstract}
RESUMO
Introdução: A retomada das atividades durante a pandemia da COVID-19 tem sido tema de discussão em diferentes países para que não afete a continuidade do atendimento às urgências e às emergências atualmente em andamento. Objetivo: Descrever as estratégias para a retomada das atividades eletivas em uma rede de hospitais públicos de ensino no Brasil durante a pandemia da COVID-19. Método: Trata-se de um estudo descritivo no formato de relato de experiência que inclui um processo de vigilância em saúde hospitalar realizado a partir da observação e discussão em uma perspectiva de rede. Resultados: 0 guia para retomada das atividades eletivas foi estruturado em quatro tópicos, sendo o segundo tópico dividido em outros 11 para detalhamento das recomendações a serem seguidas. As estratégias e ações apresentadas neste guia foram categorizadas em etapas da gestão de projetos para melhor visualização do caminho a ser percorrido: organização, planejamento, implantação e monitoramento. 0 material foi publicado em setembro de 2020 pela administração central da rede e está em fase de implantação pelos hospitais. Conclusões: 0 relato de experiência poderá auxiliar outras organizações na definição de estratégias para a retomada segura de suas atividades.
\end{abstract}

PALAVRAS-CHAVE: Infecções por Coronavírus; Vigilância em Saúde Pública; Procedimentos Cirúrgicos Eletivos

\begin{abstract}
Introduction: The resumption of activities during the COVID-19 pandemic has been a topic of discussion in different countries so that it does not affect the continuity of assistance to urgencies and emergencies currently underway. Objective: To describe strategies for resuming elective activities in a network of public teaching hospitals in Brazil during the COVID-19 pandemic. Method: This is a descriptive study in the form of an experience report that includes a hospital health surveillance process carried out based on observation and discussion from a network perspective. Results: The guide for resuming elective activities was structured into four topics, the second topic being divided into 11 others to detail the recommendations to be followed. The strategies and actions presented in this guide were categorized into project management stages to better visualize the path to be taken: organization, planning, implementation and monitoring. The material was published in September 2020 by the network's central administration and is being implemented by hospitals. Conclusions: The experience report can help other organizations to define strategies for the safe resumption of their activities.
\end{abstract}

KEYWORDS: Coronavirus Infections; Public Health Surveillance; Elective Surgical Procedures 


\section{INTRODUÇÃO}

A pandemia da COVID-19 (coronavirus disease 2019) tem desafiado os sistemas de saúde de todo o mundo a reorganizarem processos, práticas e estruturas para o enfrentamento e contenção da disseminação do novo coronavírus SARS-CoV-2, sem prejuízo ao atendimento das necessidades de saúde da população ${ }^{1,2}$.

Em um primeiro momento, considerando a alta transmissibilidade e infectividade do SARS-Cov-2, o contexto de transmissão comunitária instaurado em muitos territórios e o pouco conhecimento limitado sobre o tratamento da doença, algumas atividades assistenciais eletivas foram reprogramadas nos contextos hospitalares brasileiros ${ }^{3,4,5}$.

Essa ação teve o intuito de minimizar o risco de exposição de pacientes e profissionais de saúde ao vírus, além de permitir a concentração de esforços, materiais e estruturas no tratamento de casos emergenciais e adequações para atendimento ao novo perfil e demanda assistenciais ${ }^{2}$.

A reprogramação das atividades eletivas assistenciais no Brasil ampara-se nas recomendações da Agência Nacional de Vigilância Sanitária (Anvisa) ${ }^{6}$, associações médicas e sociedades científi$\operatorname{cas}^{4,7}$ e autoridades nacional e locais ${ }^{8}$, que também estão pautadas nas diretrizes do Ministério da Saúde do Brasil e da Organização Mundial da Saúde (OMS) ${ }^{5}$.

Os procedimentos cirúrgicos e consultas foram avaliados pelas equipes dos hospitais quanto a sua criticidade e urgência em comparação com o potencial risco de contaminação pela COVID19, de forma que pudessem ser realizados todos os procedimentos que fossem imprescindíveis no momento, dando prioridade àqueles classificados como tempo-sensíveis, tais como cirurgias de urgência ou emergência, oncológicas e cardiovasculares.

O elenco dos procedimentos eletivos assistenciais que foram represados ou adiados no contexto hospitalar brasileiro envolveu apenas aqueles que poderiam ser remarcadas sem prejuízo e danos à saúde dos pacientes, priorizando atendimentos para algumas condições, como o tratamento inicial de transtornos graves ou com risco de vida ${ }^{10}$. No âmbito específico dos hospitais universitários federais, esses procedimentos incluíram consultas ambulatoriais, cirurgias eletivas, procedimentos endoscópicos e atendimentos odontológicos, bem como estágios de graduações em saúde e aulas de residências.

A redução no atendimento eletivo não ocorreu tão somente por iniciativa ou necessidade das instituições hospitalares, mas soma-se ao fato de, até o momento (setembro de 2020), inexistir tratamento farmacológico comprovadamente efetivo para a COVID-19 e isso tem gerado receios em muitos pacientes que, consequentemente, passaram a evitar comparecer a ambientes clínicos e hospitalares².

Entretanto, o retardo no início ou na continuidade do tratamento de pacientes com doenças não emergenciais pode resultar em aumento da morbimortalidade e, a médio e longo prazo, também pode refletir no sistema de saúde que possivelmente atenderá mais pacientes com necessidade de tratamentos especializados e prolongados ${ }^{2}$.

Dessa forma, passada a fase inicial de adaptação à nova realidade assistencial e, considerando que o período da pandemia tem se prolongado por meses e ainda sem previsão de término, mostra-se relevante o estabelecimento de ações de planejamento de forma ampla e dinâmica com a finalidade de garantir o retorno das atividades eletivas em etapas para atendimento das demandas de rotina e reprimida dos pacientes que deixaram de ser atendidos devido à pandemia, com medidas necessárias à segurança do paciente e dos trabalhadores em saúde ${ }^{2}$. Essa temática tem sido alvo de estudos em diversos países e em diferentes instituições brasileiras, demonstrando a importância da retomada das atividades eletivas com planos estruturados ${ }^{11,12,13}$.

No Brasil, até 31 de julho de 2020, 21 estados brasileiros possuíam planos de flexibilização de medidas de distanciamento social implementados, os quais consideram como critérios a evolução da epidemia e a taxa de leitos ocupados ${ }^{14}$.

Nesse âmbito, para uma flexibilização e retomada segura de atividades eletivas, destaca-se a importância da vigilância em saúde para a saúde pública e da prevenção da transmissão intra-hospitalar de COVID-19.

Destaca-se, nesse sentido, as atuações transversais de vigilância em saúde hospitalar que englobam a articulação de ações intersetoriais, vigilância epidemiológica, vigilância sanitária e vigilância em saúde do trabalhador ${ }^{15}$ nos diferentes serviços do hospital. As boas práticas na prestação de serviços de saúde reduzem os riscos e propiciam a segurança do paciente e dos trabalhadores, inclusive os de saúde ${ }^{15}$.

O objetivo deste estudo foi descrever as estratégias para a retomada das atividades eletivas em uma rede de hospitais públicos de ensino no Brasil diante da pandemia da COVID-19. Buscou-se apresentar a estrutura do guia construído e as etapas planejadas para que esta ação seja conduzida sem afetar a continuidade do atendimento às urgências e emergências atualmente em andamento.

Assim, no presente estudo, consideram-se atividades assistenciais eletivas aquelas que podem ser adiadas ou não serem realizadas sem dano ao paciente. Tal consideração traz referências do conceito de procedimentos cirúrgicos eletivos que incluem cirurgias "para corrigir problemas médicos que não ameaçam a vida e também para aliviar afecções causadoras de estresse psicológico ou outro risco potencial a pacientes, por exemplo, cirurgia estética ou contraceptiva” ${ }^{16}$.

\section{MÉTODO}

Trata-se de relato de experiência que aborda a construção de estratégias para retomada das atividades eletivas em uma rede de hospitais de ensino no Brasil durante a pandemia da COVID-19. 
Refere-se a um estudo descritivo que inclui um processo de vigilância em saúde hospitalar realizado a partir da observação e discussão das estratégias de enfrentamento à pandemia de COVID-19 em uma perspectiva de rede.

Nos momentos de crise, a sistematização e o compartilhamento de experiências construídas em diferentes cenários fornecem mais subsídios para replicação e discussão em busca da contenção da disseminação do novo coronavírus e fortalecimento do Sistema Único de Saúde (SUS) 1 .

O trabalho foi elaborado em etapas durante os meses de junho, julho, agosto e setembro de 2020, contando com contribuições da rede de hospitais por meio da Câmara Técnica de Vigilância em Saúde (CTVS) (Figura). Esta Câmara foi constituída em março do ano em curso e tem como uma de suas responsabilidades o apoio à promoção de ações de vigilância em saúde e à segurança do paciente nos hospitais da rede relativas a eventos de saúde pública.

Na primeira etapa, a definição de escopo e do tipo de documento foi discutida em reuniões da CTVS conduzidas no aplicativo Teams $®$.

Para elaboração da minuta do guia na segunda etapa, foi realizado levantamento de publicações referentes ao tema nos endereços eletrônicos da OMS, dos Ministérios da Saúde e da Educação, da Anvisa, do Colégio Brasileiro de Cirurgiões (CBC) e das Sociedades Brasileiras de Anestesiologia e de Endoscopia Digestiva. Esse levantamento e a construção da minuta foram realizados pela equipe de Gestão da Qualidade da administração central da rede de hospitais.

A minuta foi, então, enviada para a CTVS para contribuições, as quais foram analisadas posteriormente e incluídas. A validação, a aprovação da versão final e a publicação do guia ocorreram em setembro de 2020.

\section{RESULTADOS E DISCUSSÃO}

O Guia para retomada das atividades eletivas durante a pandemia de COVID-19 foi estruturado em quatro tópicos, sendo o segundo tópico, referente às recomendações, dividido em outros 11 para detalhamento das ações a serem seguidas para retomada segura de cada atividade (Quadro 1). Nos apêndices do guia, foram disponibilizados modelos de formulários para triagem de sintomas respiratórios de pacientes no pré-operatório e internados, bem como de seus acompanhantes.

Por se tratar de hospitais de ensino e dada sua complexidade de relações, as orientações incluíram não só atividades assistenciais, mas também práticas de ensino e administrativas.

As estratégias para retomada das atividades eletivas descritas no guia podem ser categorizadas em quatro etapas típicas da gestão de projetos: organização, planejamento, implantação e monitoramento. Apesar de o guia não apresentar esta estrutura em etapas, esta categorização auxilia na clarificação do caminho a ser percorrido pela equipe dos hospitais (Quadro 2).

A primeira etapa envolve a organização, na qual a equipe de planejamento deve ser definida. Faz-se importante que esta equipe seja multidisciplinar e envolva os diferentes setores e instâncias do hospital. Ela será responsável por discutir as estratégias para retomada das atividades eletivas na instituição, elaborar o plano de ação, auxiliar na sua implantação e monitorá-lo.

A segunda etapa refere-se ao planejamento e inclui ações de vigilância epidemiológica, avaliação interna do hospital, identificação de casos e serviços prioritários a serem retomados, definição de critérios, fluxos e responsabilidades.

Nesta etapa, as orientações nacionais e o plano de retomada responsável das Secretarias Municipal e Estadual de Saúde de onde se situa o hospital devem ser considerados visto que a ocorrência da COVID-19 não tem uma distribuição uniforme no país9. 0 momento para a retomada das atividades eletivas deve ocorrer, preferencialmente, após análise epidemiológica com a redução sustentada na taxa de novos casos da COVID-19 durante, pelo menos, 14 dias consecutivos na área geográfica de Base Populacional.

Além disso, devem ser realizadas análises da capacidade hospitalar, como ocupação de leitos, disponibilidade de profissionais

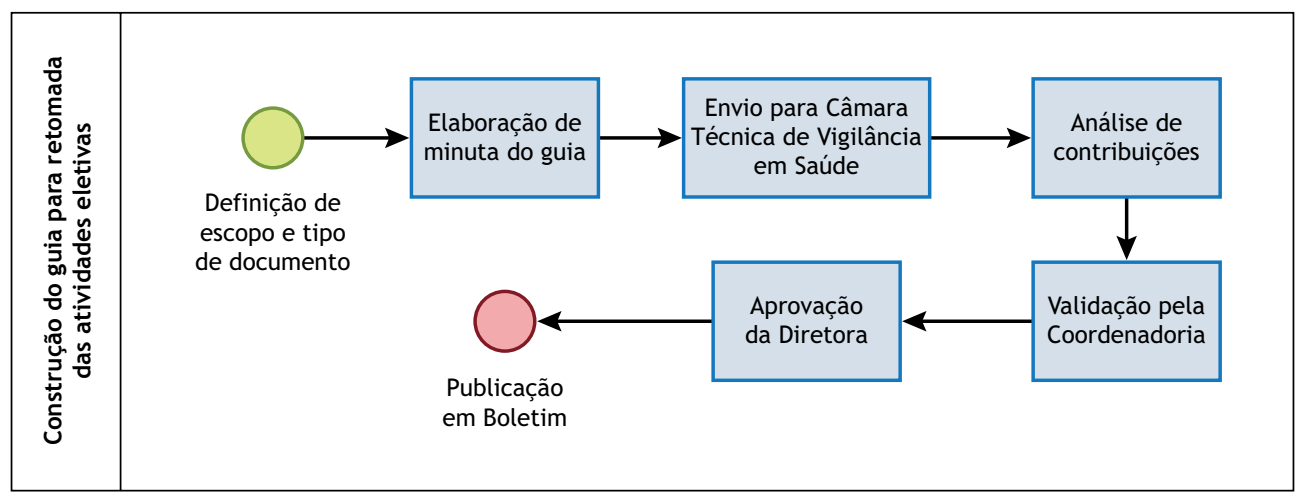

Fonte: Elaborada pelos autores, 2020.

Figura. Processo de construção do Guia para retomada das atividades eletivas na rede de hospitais de ensino durante a pandemia da COVID-19. 
Quadro 1. Estrutura do guia para retomada das atividades eletivas na rede de hospitais de ensino durante a pandemia da COVID-19.

\begin{tabular}{|c|c|c|c|c|c|}
\hline 1. & \multicolumn{5}{|c|}{ Introdução e Objetivo } \\
\hline \multirow{14}{*}{2.} & \multirow{14}{*}{ Recomendações } & 2.1 & \multicolumn{3}{|r|}{ Cuidados Gerais } \\
\hline & & 2.2. & \multicolumn{3}{|r|}{ Controle Universal da Fonte } \\
\hline & & 2.3. & \multicolumn{3}{|c|}{ Atendimento de Urgência e Emergência } \\
\hline & & 2.4. & \multicolumn{3}{|r|}{ Consultas Ambulatoriais } \\
\hline & & 2.5. & \multicolumn{3}{|c|}{$\begin{array}{l}\text { Acompanhamento multiprofissional de pacientes internados e atividades administrativas em áreas de } \\
\text { internação }\end{array}$} \\
\hline & & \multirow{4}{*}{2.6.} & \multirow{4}{*}{ Cirurgias Eletivas } & 2.6.1. & Pré-Operatório \\
\hline & & & & 2.6.2. & Intraoperatório \\
\hline & & & & 2.6.3. & Pós-Operatório \\
\hline & & & & 2.6.4. & Testagem de pacientes para procedimentos cirúrgicos \\
\hline & & 2.7. & \multicolumn{3}{|c|}{$\begin{array}{c}\text { Fortalecimento da realização de teleconsultas para atendimento a pacientes ambulatoriais e } \\
\text { acompanhamento de pacientes cirúrgicos }\end{array}$} \\
\hline & & 2.8. & \multicolumn{3}{|c|}{ Atividades de Ensino, Pesquisa e Extensão } \\
\hline & & 2.9. & \multicolumn{3}{|r|}{ Medidas Internas } \\
\hline & & 2.10. & \multicolumn{3}{|c|}{$\begin{array}{l}\text { Planejamento de estoques de produtos para saúde, incluindo fornecimento de equipamentos de } \\
\text { proteção individual (EPI) adequados para toda a equipe do serviço de saúde, e de medicamentos }\end{array}$} \\
\hline & & 2.11. & \multicolumn{3}{|c|}{ Monitoramento da retomada das atividades } \\
\hline 3. & \multicolumn{5}{|l|}{ Expectativa } \\
\hline 4. & \multicolumn{5}{|l|}{ Referências } \\
\hline- & \multicolumn{5}{|c|}{ Apêndice A - Checklist para triagem de sintomas respiratórios } \\
\hline- & \multicolumn{5}{|c|}{ Apêndice B - Checklist pré-operatório para triagem de sintomas respiratórios } \\
\hline
\end{tabular}

Fonte: Elaborado pelos autores, 2020.

de saúde, insumos (medicamentos, produtos para saúde e equipamentos), exames e procedimentos para atender a demanda assistencial. Registre-se que a falta de anestésicos é realidade constada e de conhecimento geral relatada pela Anvisa e pelo Ministério da Saúde diante do consumo e das dificuldades de produção enfrentadas pela indústria farmacêutica durante a pandemia ${ }^{17}$. Portanto, é imprescindível que este cenário seja estudado, bem como sejam avaliadas alternativas terapêuticas, caso necessárias.

A disponibilidade de testes RT-PCR para testagem de profissionais e pacientes e a infraestrutura local instalada no hospital para realização destes testes também são pontos a serem considerados neste processo. Neste momento, não se recomenda trabalhar com testes rápidos ou testes sorológicos para definir quais profissionais poderão participar das atividades eletivas e nem testagem de rotina para profissionais assintomáticos².

No planejamento, também deve-se levar em conta as atividades a serem priorizadas no plano de retomada. A orientação é iniciar pelas atividades que agreguem menor risco de transmissão da COVID-19 e que possuam maior impacto, prevendo a adoção de medidas para redução do risco de transmissão da COVID-19 e garantindo a resolubilidade da demanda de rotina, bem como a demanda reprimida.

A retomada das atividades eletivas suspensas deve ocorrer de forma gradual para que o risco de contaminação intra-hospitalar e ambulatorial seja diminuído e a segurança do paciente e do trabalhador sejam preservadas. As medidas de biossegurança devem ser aprimoradas, considerando, sobretudo, a possibilidade de transmissão da COVID-19.

Toda a programação cirúrgica deve ser avaliada em relação aos riscos, prioridades e recursos considerando ainda que: (a) pacientes com COVID-19 apresentam maior morbimortalidade no período pós-operatório, sendo que análise recente demonstrou que $44,1 \%$ dos pacientes necessitaram de UTI e a taxa de mortalidade após admissão na UTI foi de 20,5\%; (b) otimização da alocação de recursos e espaços, principalmente leitos de terapia intensiva, para a demanda decorrente da pandemia 9 .

Faz-se importante definir um cronograma do retorno das atividades ambulatoriais, apoio diagnóstico e atividades cirúrgicas em diversas especialidades orientando a sequência de retorno das atividades com a previsão de datas. Frisa-se a importância de se ter cautela nos prazos estabelecidos levando-se em consideração os riscos associados aos pacientes e profissionais de saúde em um contexto de saúde pública.

A terceira etapa trata da implantação do plano de ação e envolve como estratégias a comunicação e educação, a adequação de práticas e fluxos.

O monitoramento é a quarta e última etapa e inclui a vigilância em saúde como estratégia para o plano de retomada das atividades eletivas. Dada a incidência ainda crescente da COVID-19 em muitas cidades e regiões brasileiras, a retomada das atividades eletivas pode conduzir ao aumento do número de casos e internações, sendo necessário monitoramento constante para 
Quadro 2. Estratégias para retomada das atividades eletivas segundo etapas.

\begin{tabular}{|c|c|c|c|}
\hline Etapa & Estratégia & Ação & Observações \\
\hline \multirow{2}{*}{ 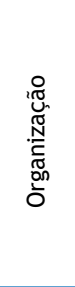 } & $\begin{array}{l}\text { Definição de equipe de } \\
\text { planejamento }\end{array}$ & $\begin{array}{c}\text { Instituição de Grupo de Trabalho de } \\
\text { Planejamento da Retomada das Atividades } \\
\text { Eletivas ou delegação de responsabilidade } \\
\text { para o COE }\end{array}$ & $\begin{array}{c}\text { Composição mínima: representantes da Superintendência, Gerências } \\
\text { Administrativa, de Ensino e Pesquisa, de Atenção à Saúde, Divisões } \\
\text { de Enfermagem, Médica, Unidades de Cirurgia, e de Gestão do } \\
\text { Atendimento Ambulatorial, Setores de Regulação e Avaliação em } \\
\text { Saúde, de Gestão da Qualidade e Vigilância em Saúde, de Suprimentos } \\
\text { e de Farmácia }\end{array}$ \\
\hline & $\begin{array}{l}\text { Elaboração de plano } \\
\text { de ação }\end{array}$ & $\begin{array}{l}\text { Elaboração em um ou mais documentos, } \\
\text { seguindo as etapas do processo de } \\
\text { retomada }\end{array}$ & $\begin{array}{l}\text { Conteúdo conforme informações deste quadro. Incluir cronograma para } \\
\text { retomada gradual }\end{array}$ \\
\hline \multirow{23}{*}{ 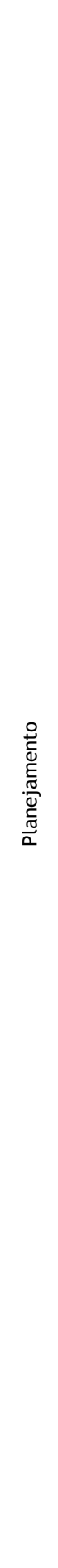 } & \multirow{5}{*}{$\begin{array}{l}\text { Vigilância } \\
\text { epidemiológica }\end{array}$} & \multirow{4}{*}{$\begin{array}{l}\text { Avaliação do cenário epidemiológico do } \\
\text { estado e município }\end{array}$} & Capacidade de resposta da RAS \\
\hline & & & Aspectos socioeconômicos e culturais dos territórios \\
\hline & & & Orientações emitidas pelas autoridades locais e órgãos de saúde \\
\hline & & & $\begin{array}{c}\text { Análise epidemiológica: redução sustentada na taxa de novos casos de } \\
\text { COVID-19 durante, pelo menos, } 14 \text { dias consecutivos na área geográfica } \\
\text { de Base Populacional }\end{array}$ \\
\hline & & Avaliação do cenário no hospital & Número de novos casos e de óbitos \\
\hline & \multirow{12}{*}{$\begin{array}{l}\text { Avaliação interna do } \\
\text { hospital }\end{array}$} & \multirow{2}{*}{$\begin{array}{l}\text { Levantamento do perfil assistencial } \\
\text { existente }\end{array}$} & $\begin{array}{l}\text { Oferta de consultas, exames e procedimentos para repactuação com os } \\
\text { gestores de novos quantitativos a serem atendidos de forma gradativa }\end{array}$ \\
\hline & & & $\begin{array}{c}\text { Aprimoramento da proteção de pacientes clinicamente mais } \\
\text { vulneráveis à COVID-19 }\end{array}$ \\
\hline & & \multirow{2}{*}{ Verificação dos recursos disponíveis } & $\begin{array}{c}\text { Disponibilidade de insumos: saneantes, medicamentos, produtos para } \\
\text { saúde, incluindo EPI e equipamentos }\end{array}$ \\
\hline & & & $\begin{array}{l}\text { Previsão de disponibilidade de testes validados e adequados (para } \\
\text { pacientes e profissionais) }\end{array}$ \\
\hline & & \multirow{4}{*}{$\begin{array}{l}\text { Análise da infraestrutura para as } \\
\text { atividades }\end{array}$} & $\begin{array}{c}\text { Estrutura adequada para higienização das mãos com água e sabonete } \\
\text { líquido ou preparação alcoólica }\end{array}$ \\
\hline & & & $\begin{array}{l}\text { Barreiras físicas para reduzir a exposição ao vírus SARS-CoV-2, como } \\
\text { janelas e barreiras de vidro ou plástico (Áreas de triagem, recepção, } \\
\text { balcão de atendimento no pronto-socorro ou na janela da farmácia) }\end{array}$ \\
\hline & & & $\begin{array}{l}\text { Infraestrutura tecnológica necessária à consulta virtual/remota e à } \\
\text { segurança da informação (equipamentos nos consultórios, sistemas } \\
\text { funcionantes e interligados) }\end{array}$ \\
\hline & & & $\begin{array}{c}\text { Sala de espera e salas de aula: distância mínima de } 1 \text { metro entre as } \\
\text { cadeiras, ventilação adequada }\end{array}$ \\
\hline & & $\begin{array}{l}\text { Avaliação da disponibilidade de pessoal } \\
\text { proporcional ao aumento de volume de } \\
\text { trabalho }\end{array}$ & $\begin{array}{l}\text { Considerar equipe assistencial, serviço de limpeza, engenharia, } \\
\text { processamento e esterilização dos materiais e equipamentos etc. }\end{array}$ \\
\hline & & \multirow{3}{*}{ Análise da capacidade hospitalar } & Número de leitos ocupados \\
\hline & & & $\begin{array}{l}\text { Disponibilidade de leitos nas áreas destinadas para coorte de pacientes } \\
\text { suspeitos e confirmados de COVID-19, de leitos de UTI e equipamentos } \\
\text { de suporte à vida }\end{array}$ \\
\hline & & & $\begin{array}{l}\text { Disponibilidade de serviços de apoio: central de exames de imagem, } \\
\text { patologia, capacidade de testagem para COVID-19 dos laboratórios }\end{array}$ \\
\hline & \multirow{3}{*}{$\begin{array}{l}\text { Identificação de casos } \\
\text { e serviços prioritários } \\
\text { a serem retomados }\end{array}$} & $\begin{array}{l}\text { Avaliação da urgência do atendimento ou } \\
\text { intervenção e do risco associado }\end{array}$ & $\begin{array}{c}\text { Utilização da matriz } 2 \times 2 \text { e outras ferramentas e documentos. } \\
\text { Verificação do potencial de necessidade de leitos em UTI no pós- } \\
\text { operatório }\end{array}$ \\
\hline & & Identificação de recursos requeridos & $\begin{array}{l}\text { Uso de sangue e derivados, medicamentos e produtos para a saúde } \\
\text { disponíveis }\end{array}$ \\
\hline & & Definição de prioridades & $\begin{array}{l}\text { Listas de espera cirúrgica; de casos cancelados e adiados } \\
\text { anteriormente; priorização de especialidades (oncologia, transplante, } \\
\text { trauma, cardíaca etc.) ou de pacientes em sofrimento (por exemplo: } \\
\text { dor severa). Relação com os serviços contratualizados com o gestor do } \\
\text { SUS }\end{array}$ \\
\hline & \multirow[t]{2}{*}{ Definição de critérios } & De admissão de pacientes & $\begin{array}{c}\text { Pacientes que permanecerem assintomáticos e em quarentena por } 14 \\
\text { dias antes da admissão e, quando possível, apresentarem resultado } \\
\text { negativo do teste RT-PCR antes da admissão em período determinado } \\
\text { pela equipe da CCIRAS do hospital }\end{array}$ \\
\hline & & De classificação de risco & Para identificação e pronto atendimento dos casos \\
\hline & Definição de fluxos & $\begin{array}{l}\text { Disponibilização de protocolos, fluxos e } \\
\text { instruções de trabalho ou Procedimentos } \\
\text { Operacionais Padrões (POP) }\end{array}$ & $\begin{array}{c}\text { Para identificação, medidas de prevenção e controle de infecção, } \\
\text { entre outras atividades. Obrigatoriedade dos termos de consentimento } \\
\text { de cirurgia e anestesia, com ciência de que o procedimento será } \\
\text { realizado durante a pandemia de COVID-19 }\end{array}$ \\
\hline
\end{tabular}


continuação

\begin{tabular}{|c|c|c|c|}
\hline Etapa & Estratégia & Ação & Observações \\
\hline \multirow{2}{*}{ 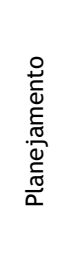 } & \multirow{2}{*}{$\begin{array}{l}\text { Definição de } \\
\text { responsabilidades }\end{array}$} & $\begin{array}{l}\text { Designar funcionários responsáveis por } \\
\text { identificar situações potencialmente } \\
\text { perigosas no intuito de agir rapidamente } \\
\text { para mitigação dos riscos }\end{array}$ & $\begin{array}{l}\text { Por exemplo: falhas nos procedimentos de biossegurança, uso ou } \\
\text { descarte de EPI }\end{array}$ \\
\hline & & $\begin{array}{l}\text { Definição de coorte de profissionais em } \\
\text { áreas de pacientes com COVID-19 e de } \\
\text { suspeitos }\end{array}$ & $\begin{array}{l}\text { A equipe de profissionais que atua na assistência de pacientes com } \\
\text { COVID-19 deve ser preferencialmente diferente da que atua com } \\
\text { pacientes suspeitos de COVID-19, bem como distinta da que atua no } \\
\text { atendimento a outros pacientes }\end{array}$ \\
\hline \multirow{27}{*}{ 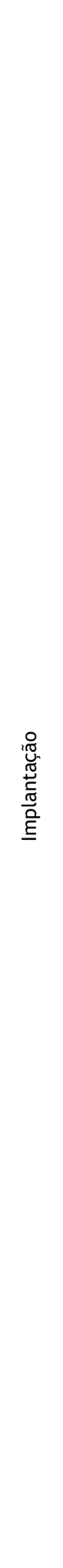 } & \multirow{6}{*}{$\begin{array}{l}\text { Comunicação e } \\
\text { Educação }\end{array}$} & Divulgação do Plano de Retomada & Para todos os profissionais e alunos \\
\hline & & \multirow{3}{*}{$\begin{array}{l}\text { Reforço às campanhas de conscientização } \\
\text { e estratégias de comunicação visual }\end{array}$} & Uso obrigatório de máscara de proteção \\
\hline & & & Higienização das mãos \\
\hline & & & Distanciamento social \\
\hline & & $\begin{array}{l}\text { Capacitação continuada dos trabalhadores } \\
\text { e profissionais de saúde }\end{array}$ & $\begin{array}{l}\text { Incluir todas as etapas do processo (por exemplo: recepção, } \\
\text { higienização do ambiente, identificação de casos suspeitos etc.) }\end{array}$ \\
\hline & & $\begin{array}{l}\text { Marcações e sinalizações para auxiliar na } \\
\text { manutenção do distanciamento } \\
\text { entre profissionais, pacientes } \\
\text { e acompanhantes }\end{array}$ & Placas de acrílico, faixa no piso etc. \\
\hline & \multirow{14}{*}{ Adequação de práticas } & \multirow{6}{*}{$\begin{array}{l}\text { Organização e agendamento das } \\
\text { atividades (otimização da agenda quanto } \\
\text { a horário, sala e equipe) }\end{array}$} & $\begin{array}{l}\text { Consultas em sequência do mesmo paciente para o mesmo dia nas } \\
\text { diferentes especialidades }\end{array}$ \\
\hline & & & $\begin{array}{l}\text { Flexibilização da agenda: horários e dias alternativos dos usuais } \\
\text { (horários antecipados, fins de semana, horários noturnos) }\end{array}$ \\
\hline & & & Espaçamento maior de horário entre pacientes \\
\hline & & & Reagendamento de consultas de pacientes sintomáticos respiratórios \\
\hline & & & Não realizar mutirões \\
\hline & & & $\begin{array}{l}\text { Avaliação do estado de saúde do paciente no pré-operatório, por } \\
\text { teleatendimento }\end{array}$ \\
\hline & & $\begin{array}{l}\text { Consultas ambulatoriais virtuais remotas e } \\
\text { multiprofissionais }\end{array}$ & \multirow[t]{2}{*}{ Predefinição de casos e perfis elegíveis a esse tipo de atendimento } \\
\hline & & Telemedicina na avaliação pós-operatória & \\
\hline & & $\begin{array}{l}\text { Reuniões de equipe multidisciplinares } \\
\text { virtuais }\end{array}$ & Utilização de plataformas digitais institucionalmente estabelecidas \\
\hline & & \multirow{2}{*}{$\begin{array}{l}\text { Reavaliação do tempo de permanência } \\
\text { hospitalar }\end{array}$} & Alta precoce para pacientes com sintomas leves \\
\hline & & & $\begin{array}{l}\text { Acompanhamento domiciliar para aqueles que não necessitam de } \\
\text { internação }\end{array}$ \\
\hline & & \multirow[b]{2}{*}{ Planejamento de atividades à beira-leito } & $\begin{array}{l}\text { Agrupamento de atividades para minimizar o número de entradas no } \\
\text { quarto, área e box do paciente }\end{array}$ \\
\hline & & & $\begin{array}{l}\text { Avaliação criteriosa da prescrição medicamentosa (principalmente em } \\
\text { relação aos horários), rotina de coleta e realização de exames diários e } \\
\text { da prescrição de cuidados }\end{array}$ \\
\hline & & Intervalos de equipe diferenciados & $\begin{array}{l}\text { Limitar a densidade de profissionais em áreas específicas, como, } \\
\text { vestiários, áreas de repouso, copas e refeitórios }\end{array}$ \\
\hline & \multirow{7}{*}{ Adequação de fluxos } & $\begin{array}{l}\text { Adequação dos fluxos e processos } \\
\text { associados às atividades eletivas }\end{array}$ & Redução do fluxo de funcionários e pacientes \\
\hline & & $\begin{array}{l}\text { Limitação de pontos de entrada ao } \\
\text { hospital, de horários de visita e de } \\
\text { acompanhantes }\end{array}$ & $\begin{array}{l}\text { Estratégias para maior controle e triagem de sintomas respiratórios e } \\
\text { redução de fluxo de pessoas }\end{array}$ \\
\hline & & \multirow{2}{*}{$\begin{array}{l}\text { Triagem de sintomáticos respiratórios em } \\
\text { pacientes, acompanhantes e visitantes }\end{array}$} & Formulários de checagem \\
\hline & & & Aferição da temperatura \\
\hline & & $\begin{array}{l}\text { Isolamento imediato de pacientes com } \\
\text { diagnóstico positivo ou que apresente } \\
\text { sintomas após admissão }\end{array}$ & $\begin{array}{l}\text { Fornecer máscaras cirúrgicas na entrada do pronto atendimento para } \\
\text { pacientes com sintomas respiratórios }\end{array}$ \\
\hline & & $\begin{array}{l}\text { Ampliação da realização de testes para } \\
\text { COVID-19 }\end{array}$ & Para pacientes e profissionais sempre que possível \\
\hline & & $\begin{array}{l}\text { Intensificação da limpeza e desinfecção do } \\
\text { ambiente hospitalar e de ambulância }\end{array}$ & Recomendações da OMS por tipo de área e da Anvisa \\
\hline
\end{tabular}

continua 
continuação

\begin{tabular}{|c|c|c|c|}
\hline Etapa & Estratégia & Ação & Observações \\
\hline \multirow{6}{*}{ 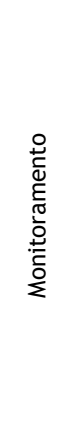 } & \multirow{6}{*}{ Vigilância em saúde } & $\begin{array}{l}\text { Vigilância de soroprevalência e } \\
\text { soroconversão }\end{array}$ & Inclusão de pacientes e equipe de profissionais \\
\hline & & $\begin{array}{l}\text { Vigilância de eventos adversos ao longo } \\
\text { do tempo }\end{array}$ & $\begin{array}{l}\text { Planejamento de ações para recuar nos atendimentos eletivos caso } \\
\text { necessário }\end{array}$ \\
\hline & & $\begin{array}{c}\text { Acompanhamento da publicação de novas } \\
\text { normativas e atualizações }\end{array}$ & $\begin{array}{l}\text { Verificação diária e contínua de resoluções e notas técnicas da Anvisa, } \\
\text { Ministério da Saúde, Secretarias Estaduais e Municipais de Saúde }\end{array}$ \\
\hline & & $\begin{array}{l}\text { Vigilância ativa e passiva dos } \\
\text { colaboradores do hospital }\end{array}$ & $\begin{array}{c}\text { Avaliação diária e contínua para identificação de sintomas de suspeição } \\
\text { para COVID-19 (aferição de temperatura, questionários) }\end{array}$ \\
\hline & & $\begin{array}{l}\text { Análise de indicadores específicos da } \\
\text { COVID-19 }\end{array}$ & $\begin{array}{l}\text { Avaliação do cenário epidemiológico na área geográfica de Base } \\
\text { Populacional }\end{array}$ \\
\hline & & $\begin{array}{l}\text { Acompanhamento da disponibilidade de } \\
\text { recursos }\end{array}$ & Medicamentos, insumos e produtos para a saúde \\
\hline
\end{tabular}

Fonte: Elaborado pelos autores, a partir das informações do Guia para Retomada das atividades eletivas durante a pandemia de COVID-19, 2020.

COE: Centro de Operações de Emergências; RAS: Rede de Atenção à Saúde; EPI: equipamento de proteção individual; UTI; unidade de terapia intensiva; CCIRAS: Comissão de Controle de Infecção Relacionada à Assistência à Saúde; OMS; Organização Mundial da Saúde; Anvisa: Agência Nacional de Vigilância Sanitária.

que ações de recuada possam ser implantadas rapidamente caso necessário ${ }^{15}$. Os efeitos só serão percebidos cerca de duas semanas após implantação, e, caso sejam necessárias adequações, um período equivalente será necessário para que os resultados sejam evidenciados ${ }^{15}$.

\section{CONCLUSÕES}

As estratégias para a retomada das atividades eletivas em uma rede de hospitais públicos de ensino no Brasil diante da pandemia da COVID-19 foram descritas neste estudo, bem como a estrutura do guia construído. As estratégias planejadas foram categorizadas conforme etapas da gestão de projetos para melhor compreensão de como esta ação pode ser conduzida sem afetar a continuidade do atendimento às urgências e às emergências atualmente em andamento nos hospitais de ensino do país.
Verificou-se a importância das ações de vigilância em saúde hospitalar nas diferentes etapas do plano de retomada.

No âmbito do trabalho desta rede de hospitais, as próximas etapas incluem: o apoio da gestão para o processo de construção dos Planos de Ação; a implantação e o monitoramento do processo de retomada das atividades eletivas durante a pandemia da COVID-19.

Dada a diversidade de hospitais existentes na rede e de cenários epidemiológicos, cada hospital deve elaborar e manter atualizado seu plano de ação de retomada das atividades eletivas, considerando as variáveis que interferem em sua realidade.

O relato desta experiência poderá auxiliar outras organizações na definição de estratégias para a retomada segura de suas atividades sem prejuízo à assistência e saúde da população.

\section{REFERÊNCIAS}

1. Ribeiro MA, Araújo Júnior DG, Cavalcante ASP, Martins AF, Sousa LA, Carvalho RC et al. (Re)organização da atenção primária à saúde para o enfrentamento da COVID-19: experiência de Sobral CE. Aps Rev. 2020;2(2):177-88. https://doi.org/10.14295/aps.v2i2.125

2. Empresa Brasileira de Serviços Hospitalares - Ebserh. Guia para retomada das atividades eletivas durante a pandemia da COVID-19. Boletim de Serviço 896. 4 set 2020[acesso 26 set 2020]. Disponível em: https://www.gov.br/ebserh/ pt-br/acesso-a-informacao/boletim-de-servico/sede/2020/ boletim_servico_896_04_09_2020.pdf

3. Silva LED, Cohen RV, Andrade JC, Szegö T, Santo MA, Ramos $\mathrm{AC}$ et al. The recommendations of the brazilian college of surgeons and the brazilian bariatric and metabolic surgery societies on the return of bariatric and metabolic operations in geographic regions of the country where the procedures have been allowed by local policies, in the period of COVID-19 pandemic. Rev Col Bras Cir. 2020;47:1-10. https://doi.org/10.1590/0100-6991e-20202640
4. Silva LE, Cohen RV, Rocha JLL, Hassel VMC, Von-Bahten LC. Elective surgeries in the new normal post-COVID-19 pandemic: to test or do not test? Rev Col Bras Cir. 2020;47:1-7. https://doi.org/10.1590/0100-6991e-20202649

5. Pan American Health Organization - PAHO. Reorganização e expansão progressiva dos serviços de saúde para resposta à pandemia de COVID-19. Washington: Pan American Health Organization; 2020[acesso 25 ago 2020]. Disponível em: https://iris.paho.org/handle/10665.2/52068

6. Agência Nacional de Vigilância Sanitária - Anvisa. Orientações para serviços de saúde: medidas de prevenção e controle que devem ser adotadas durante a assistência aos casos suspeitos ou confirmados de infecção pelo novo coronavírus (SARS-CoV-2). Brasília: Agência Nacional de Vigilância Sanitária; 2020[acesso 25 ago 2020]. Disponível em: http://portal.anvisa.gov.br/documents/33852/271858/ Nota+T\%C3\%A9cnica+n+04-2020+GVIMS-GGTES-ANVISA/ ab598660-3de4-4f14-8e6f-b9341c196b28 
7. Conselho Federal de Medicina - CFM. Combate à COVID-19: recomendação aos CRMs sobre avaliação dos atendimentos eletivos. Brasília: Conselho Federal de Medicina; 2020[acesso 25 ago 2020]. Disponível em: https://portal. cfm.org.br/images/stories/pdf/recomendacao-cfmatendimentos-eletivos.pdf

8. Brasil. Lei № 14.019 , de 2 de julho de 2020. Altera a lei № 13.979, de 6 de fevereiro de 2020, para dispor sobre a obrigatoriedade do uso de máscaras de proteção individual para circulação em espaços públicos e privados acessíveis ao público, em vias públicas e em transportes públicos, sobre a adoção de medidas de assepsia de locais de acesso público, inclusive transportes públicos, e sobre a disponibilização de produtos saneantes aos usuários durante a vigência das medidas para enfrentamento da emergência de saúde pública de importância internacional decorrente da pandemia da COVID-19. Diário Oficial União. 8 set 2020.

9. Agência Nacional de Vigilância Sanitária - Anvisa. Orientações para a prevenção e o controle das infecções pelo novo coronavírus (SARS-CoV-2) em procedimentos cirúrgicos. Brasília: Agência Nacional de Vigilância Sanitária; 2020[acesso 25 ago 2020]. Disponível em: http://portal.anvisa.gov.br/documents/33852/271858/ Nota+t\%C3\%A9cnica+06-2020+GVIMS-GGTESANVISA/40edaf7d-8f4f-48c9-b876-bee0090d97ae

10. Pan American Health Organization - PAHO. Manutenção de serviços essenciais de saúde: orientação operacional para o contexto da COVID-19: orientação provisória. Washington: Pan American Health Organization; 2020[acesso 25 ago 2020]. Disponível em: https://iris.paho.org/bitstream/ handle/10665.2/52363/OPASWBRACOVID-1920083_por. pdf?sequence $=2 \&$ isAllowed $=y$

11. International Labour Organization - ILO. Safe return to work: guide for employers on COVID-19 prevention.
Geneva: International Labour Organization; 2020[acesso 25 ago 2020]. Disponível em: http://www.ilo.org/actemp/ publications/WCMS_744033/lang--en/index.htm

12. Ministério da Educação (BR). Protocolo de biossegurança para retorno das atividades nas instituições federais de ensino. Brasília: Ministério da Educação; 2020[acesso 25 ago 2020]. Disponível em: https://www.gov.br/mec/ pt-br/centrais-de-conteudo/campanhas-1/coronavirus/ CARTILHAPROTOCOLODEBIOSSEGURANAR101.pdf

13. Sociedade Brasileira de Anestesologia - SBA. Orientação para o retorno das atividades anestésico-cirúrgicas no contexto da COVID-19. Rio de Janeiro: Sociedade Brasileira de Anestesologia; 2020[acesso 25 ago 2020]. Disponível em: https: / / www.sbahq.org/orientacao-para-o-retorno-dasatividades-anestesico-cirurgicas-no-contexto-da-covid-19/

14. Moraes RF, Silva LLS, Toscano CM. COVID-19 e medidas de distanciamento social no Brasil: análise comparativa dos planos estaduais de flexibilização. Brasília: Instituto de Pesquisa Econômica Aplicada; 2020[acesso 24 ago 2020]. Disponível em: http://repositorio.ipea.gov.br/ handle/11058/10165

15. Conselho Nacional de Saúde - CNS. Plano nacional de enfrentamento à pandemia da COVID-19. Brasília: Conselho Nacional de Saúde; 2020[acesso 25 ago 2020]. Disponível em: https: / / frentepelavida.org.br

16. Biblioteca Virtual em Saúde - BVS. Descritores em ciência da saúde: server: list terms. Brasília: Biblioteca Virtual em Saúde; 2020[acesso 28 de ago de 2020]. Disponível em: http://decs.bvs.br/cgi-bin/wxis1660.exe/decsserver/

17. Conselho Nacional de Secretários de Saúde - Conass. Conass alerta para a falta de medicamentos para sedação de pacientes com COVID-19. Pfarma Coronavirus. 4 jun 2020[acesso 28 ago 2020]. Disponível em: https: / /pfarma. com.br/coronavirus/5703-falta-medicamento-covid19.html

\section{Agradecimentos}

Aos profissionais que integram a Câmara Técnica de Vigilância em Saúde que participaram ativamente na construção do guia para a retomada das atividades eletivas na rede de hospitais públicos de ensino.

Contribuição dos Autores

Silva GO, Piuvezam G - Concepção, planejamento (desenho do estudo), análise, interpretação dos dados e redação do trabalho. Sasso MAD - Concepção, planejamento (desenho do estudo), análise e interpretação dos dados. Cunha LMM, Silva TBM, Bezerra INM - Aquisição, análise e interpretação dos dados. Todos os autores aprovaram a versão final do trabalho.

Os autores informam não haver qualquer potencial conflito de interesse com pares e instituições, políticos ou financeiros deste estudo.

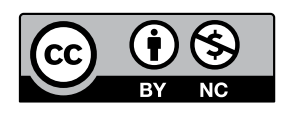

Licença CC BY-NC atribuição não comercial. Com essa licença é permitido acessar, baixar (download), copiar, imprimir, compartilhar, reutilizar e distribuir os artigos, desde que para uso não comercial e com a citação da fonte, conferindo os devidos créditos de autoria e menção à Visa em Debate. Nesses casos, nenhuma permissão é necessária por parte dos autores ou dos editores. 